Pacific

Journal of

Mathematics

INTRINSIC OBSTRUCTIONS TO THE EXISTENCE OF ISOMETRIC MINIMAL IMMERSIONS

TheOdoros Vlachos

Volume $205 \quad$ No. 2

August 2002 


\title{
INTRINSIC OBSTRUCTIONS TO THE EXISTENCE OF ISOMETRIC MINIMAL IMMERSIONS
}

\author{
Theodoros Vlachos
}

\begin{abstract}
S.S. Chern raised the problem to find necessary and sufficient conditions for a given Riemannian manifold to be realizable on a minimal submanifold of a Euclidean space. The aim of this paper is to provide new necessary conditions. For minimal submanifolds in a Euclidean space we consider the negative of the Ricci tensor as defining a new metric, which is nothing but the third fundamental form, and seek curvature properties of this metric.
\end{abstract}

\section{Introduction.}

It is well-known that the Ricci curvature of a minimal submanifold of a Euclidean space is negative semi-definite. Moreover, there are no compact minimal submanifolds in a Euclidean space. S.S. Chern [8] asked to search for further necessary conditions on those Riemannian metrics that admit an isometric minimal immersion into a Euclidean space. In this paper we deal with this question. In [10], Chern and Osserman studied the more general question, namely, to characterize those Riemannian metrics that arise as the induced metrics on minimal submanifolds of a Euclidean space. This problem has two quite different aspects, depending on whether or not one specifies the codimension. The codimension-one case, which plays as usual a prominent role, has been settled. The well-known Ricci condition provides a necessary and sufficient condition for a two-dimensional metric to be realized on a minimal surface in the three-dimensional Euclidean space $E^{3}$. Chern and Osserman [10] generalized the Ricci condition and answered the question for higher-dimensional minimal hypersurfaces. Do Carmo and Dajczer [5] gave necessary and sufficient conditions for a Riemannian metric to be minimally immersed as a hypersurface in a space form. A necessary condition for a Riemannian metric to be minimally immersed as a hypersurface in a Euclidean space was given in [1]. Chen in [7] gave a further necessary condition for a Riemannian manifold to admit an isometric minimal immersion into a Euclidean space.

The case of higher codimension presents difficulties, even in finding necessary conditions. In the special case of dimension two, and if the codimension is not specified, a complete answer to the question of Chern was given by 
Calabi [3, 4] (see also Lawson [12]). Although the result of Calabi provides necessary and sufficient conditions for the realization of a metric on a minimal surface, it would be impractical to apply it. Therefore, it is useful to have conditions that are only necessary, but are easily verified. An important example is the following result due to Barbosa and do Carmo [2]: Let $d s^{2}$ be the metric induced on a two-dimensional minimal surface in the $n$ dimensional Euclidean space $E^{n}, n \geq 3$, and let $K$ be its Gaussian curvature. Then $K \leq 0$ and whenever $K<0$, the metric $d \hat{s}^{2}=-K d s^{2}$ has Gaussian curvature $\hat{K} \leq 2$. The proof of this result relies on the holomorphicity of the Gauss map. It is worth noticing that the metric $d \hat{s}^{2}$ is nothing but the third fundamental form introduced by Obata [14].

In this paper, we deal with Chern's question in high dimension without specifying the codimension, and seek necessary conditions for a Riemannian metric to be minimally immersed in a Euclidean space, in the spirit of the above result due to Barbosa and do Carmo. We recall that for a submanifold $M^{n}$ of the Euclidean space $E^{n+p}$, the generalized Gauss map $g$ is a map whose domain is $M^{n}$, and whose range is the Grassmannian $G_{n, n+p}$ of $n$ planes in $E^{n+p}$. There is a canonical metric $d \sigma^{2}$ on the Grassmannian $G_{n, n+p}$. The metric which is induced on $M^{n}$ by $g$ is called by Obata [14] the third fundamental form of $M^{n}$, and is denoted by III, i.e., III $=g^{*}\left(d \sigma^{2}\right)$. Obata [14] proved the following formula relating III to the fundamental quantities associated with $M^{n}$

$$
\mathrm{III}=n\langle H, B\rangle-\mathrm{Ric},
$$

where $B$ is the second fundamental form of $M^{n}, H$ is the mean curvature vector, and Ric denotes the Ricci tensor. For minimal submanifolds the above formula of Obata becomes

$$
\text { III }=- \text { Ric. }
$$

From this we immediately see that the third fundamental form is intrinsic. This formula is the key tool for obtaining our results. In fact, what we are trying to do is to find curvature properties of the metric III $=-$ Ric. The question of finding curvature properties of this metric was raised by Osserman [15]. Every property of this metric one finds provides a necessary condition for a given metric to be realized on a minimal submanifold of a Euclidean space.

For any Riemannian manifold $(M,\langle\rangle$,$) with sectional curvature K$, and any point $x \in M$, we put $(\inf K)(x)=\inf \{K(\pi): 2$-plane sections $\pi \subset$ $\left.T_{x} M\right\}$. Then $\inf K$ is a well-defined function on $M$. We use this notation throughout the paper. The aim of the present paper is to prove the following local results.

Theorem A. Let $M^{n}, n \geq 3$, be an $n$-dimensional minimal submanifold of the $(n+p)$-dimensional Euclidean space $E^{n+p}$ with negative Ricci curvature. 
Then the sectional curvature $K_{\text {III }}$ of the third fundamental form III satisfies

$$
\inf K_{\text {III }}<\frac{3 n \tau^{2}-2\|\operatorname{Ric}\|^{2}-(n-1) \Delta \tau}{2\left(\tau^{2}-\|\operatorname{Ric}\|^{2}\right)},
$$

where $\tau$ is the scalar curvature (not normalized) of $M^{n}$, \|Ric\| is the length of the Ricci tensor, and $\Delta$ is the Laplacian operator of $M^{n}$.

The following corollary is an immediate consequence of Theorem A.

Corollary A. Let $M^{n}, n \geq 3$, be an $n$-dimensional Riemannian manifold with negative Ricci curvature and consider the Riemannian metric $\langle,\rangle_{*}=-$ Ric. A necessary condition for $M^{n}$ to admit an isometric minimal immersion into a Euclidean space is the following

$$
\inf K_{*}<\frac{3 n \tau^{2}-2\|\operatorname{Ric}\|^{2}-(n-1) \Delta \tau}{2\left(\tau^{2}-\|\operatorname{Ric}\|^{2}\right)},
$$

where $K_{*}$ is the sectional curvature of $\langle,\rangle_{*}, \tau$ is the scalar curvature of $M^{n}$, $\|\mathrm{Ric}\|$ is the length of the Ricci tensor, and $\Delta$ is the Laplacian operator of $M^{n}$.

For minimal submanifolds with flat normal bundle we have the following sharp result.

Theorem B. Let $M^{n}, n \geq 2$, be an $n$-dimensional minimal submanifold of the $(n+p)$-dimensional Euclidean space $E^{n+p}$ with flat normal bundle and negative Ricci curvature. Then the sectional curvature $K_{\mathrm{III}}$ of the third fundamental form III satisfies

$$
\inf K_{\text {III }} \leq 1
$$

and the equality holds if and only if $M^{n}$ lies as a minimal hypersurface in an $(n+1)$-dimensional affine subspace of $E^{n+p}$.

Corollary B. Let $M^{n}, n \geq 2$, be an n-dimensional Riemannian manifold with negative Ricci curvature and consider the Riemannian metric $\langle,\rangle_{*}=-$ Ric. A necessary condition for $M^{n}$ to admit an isometric minimal immersion with flat normal bundle into a Euclidean space is the following

$$
\inf K_{*} \leq 1,
$$

where $K_{*}$ is the sectional curvature of $\langle,\rangle_{*}$.

The paper is organized as follows: Section 2 is devoted to some notations and preliminaries. In Section 3, we derive formulas for the Riemannian connection and the curvature tensor of the third fundamental form of an arbitrary submanifold of a Euclidean space. In Section 4, we study the third fundamental form of minimal submanifolds in a Euclidean space and prove some auxiliary results. The paper ends up with Section 5, where the proofs of the main results are presented. 


\section{Preliminaries.}

Let $M^{n}$ be an $n$-dimensional submanifold of the $(n+p)$-dimensional Euclidean space $E^{n+p}$ equipped with the induced metric $\langle$,$\rangle . Denote the stan-$ dard connection of $E^{n+p}$ by $\bar{\nabla}$, the Riemannian connection of $M^{n}$ by $\nabla$, and the second fundamental form by $B$. For any tangent vector fields $X$ and $Y$ of $M^{n}$, we have the Gauss formula

$$
\bar{\nabla}_{X} Y=\nabla_{X} Y+B(X, Y)
$$

and the Weingarten formula

$$
\bar{\nabla}_{X} \xi=-A_{\xi} X+\nabla_{X}^{\frac{1}{X}} \xi
$$

where the $(1,1)$ tensor field $A_{\xi}$ is the shape operator associated with a normal vector field $\xi$, and $\nabla^{\perp}$ is the connection in the normal bundle of $M^{n}$. It is well-known that $\left\langle A_{\xi} X, Y\right\rangle=\langle B(X, Y), \xi\rangle$. Using the Gauss and Weingarten formulas, one can derive the well-known equations of Gauss, Codazzi and Ricci, which are respectively

$$
\begin{aligned}
\langle R(X, Y) Z, W\rangle=\langle & B(X, W), B(Y, Z)\rangle-\langle B(X, Z), B(Y, W)\rangle, \\
& \left(\widetilde{\nabla}_{X} A_{\xi}\right) Y=\left(\widetilde{\nabla}_{Y} A_{\xi}\right) X
\end{aligned}
$$

and

$$
R^{\perp}(X, Y) \xi=B\left(X, A_{\xi} Y\right)-B\left(A_{\xi} X, Y\right),
$$

where $R$ is the curvature tensor of $M^{n}, R^{\perp}$ is the normal curvature tensor given by

and by definition

$$
R^{\perp}(X, Y) \xi=\nabla_{X}^{\perp} \nabla_{Y}^{\perp} \xi-\nabla_{Y}^{\perp} \nabla_{X}^{\perp} \xi-\nabla_{[X, Y]}^{\perp} \xi
$$

$$
\left(\widetilde{\nabla}_{X} A_{\xi}\right) Y=\left(\nabla_{X} A_{\xi}\right) Y-A_{\nabla_{X}^{\frac{1}{X}} \xi} Y=\nabla_{X}\left(A_{\xi} Y\right)-A_{\xi}\left(\nabla_{X} Y\right)-A_{\nabla_{X}^{\perp} \xi} Y .
$$

We denote by $S$ and $\tau$ the squared length of the second fundamental form $B$ and the scalar curvature (not normalized), respectively.

We adopt the following convention on the ranges of indices:

$$
1 \leq i, j, k, \ldots, \leq n, \quad n+1 \leq \alpha, \beta, \gamma, \ldots, \leq n+p .
$$

Let $\left\{e_{1}, \ldots, e_{n}\right\}$ be a local orthonormal frame field in the tangent bundle of $M^{n}$. The mean curvature vector $H$ is defined by

$$
H=\frac{1}{n} \sum_{i} B\left(e_{i}, e_{i}\right)
$$

or equivalently

$$
H=\frac{1}{n} \sum_{\alpha}\left(\operatorname{tr} A_{\alpha}\right) e_{\alpha}
$$

where $\left\{e_{n+1}, \ldots, e_{n+p}\right\}$ is a local orthonormal frame field in the normal bundle of $M^{n}$, and $A_{\alpha}$ denotes the shape operator associated with $e_{\alpha}$. Let 
Ric be the Ricci tensor of $M^{n}$. Using the Gauss equation, we find that the Ricci tensor is given by

$$
\operatorname{Ric}(X, Y)=n\left\langle A_{H} X, Y\right\rangle-\sum_{\alpha}\left\langle A_{\alpha}^{2} X, Y\right\rangle .
$$

The Gauss map $g$, which assigns to each point $x \in M^{n}$ the $n$-plane through the origin of $E^{n+p}$ that is parallel to the tangent space of $M^{n}$ at $x$, is a map from $M^{n}$ into the Grassmannian $G_{n, n+p}=O(n+p) / O(n) \times O(p)$. There is a canonical Riemannian metric $d \sigma^{2}$ on $G_{n, n+p}$. The metric III $=g^{*}\left(d \sigma^{2}\right)$ which is induced on $M^{n}$ by $g$ is called by Obata [14] the third fundamental form of $M^{n}$, and is given by

$$
\operatorname{III}(X, Y)=n\left\langle A_{H} X, Y\right\rangle-\operatorname{Ric}(X, Y),
$$

or equivalently

$$
\operatorname{III}(X, Y)=\langle Q X, Y\rangle
$$

where $Q$ is given by

$$
Q=\sum_{\alpha} A_{\alpha}^{2}
$$

In particular, for minimal submanifolds we have

$$
\operatorname{III}(X, Y)=-\operatorname{Ric}(X, Y) .
$$

Moreover, III is intrinsic and positive definite at points where the Ricci curvature is negative.

It follows immediately from the Ricci equation that if the normal connection $\nabla^{\perp}$ is flat, then at each point there exists an orthonormal basis of the tangent space which simultaneously diagonalizes all shape operators. We use the above mentioned notation throughout the paper.

\section{Connection and curvature of the third fundamental form.}

In this section, we derive formulas for the Riemannian connection and the curvature tensor of the third fundamental form for submanifolds in a Euclidean space, not necessarily minimal.

Proposition 3.1. Let $M^{n}$ be an n-dimensional submanifold of the Euclidean space $E^{n+p}$ with positive definite third fundamental form III. Then the Riemannian connection $\nabla^{\mathrm{III}}$ of III is given by

$$
\nabla_{X}^{\mathrm{III}} Y=\sum_{\alpha} Q^{-1}\left(A_{\alpha}\left(\nabla_{X}\left(A_{\alpha} Y\right)\right)\right)-\sum_{\alpha} Q^{-1}\left(A_{\alpha}\left(A_{\nabla_{X}^{\perp} e_{\alpha}} Y\right)\right),
$$

where $X, Y$ are tangent vector fields of $M^{n}$.

We need the following auxiliary lemma. 
Lemma 3.2. Let $M^{n}$ be an $n$-dimensional submanifold of the Euclidean space $E^{n+p}$. Then the following holds

$$
\sum_{\alpha} A_{\alpha} \circ A_{\nabla \frac{1}{X} e_{\alpha}}+\sum_{\alpha} A_{\nabla_{X}^{\frac{1}{X}} e_{\alpha}} \circ A_{\alpha}=0
$$

where $X$ is a tangent vector field of $M^{n}$.

Proof. Let $\omega_{\alpha \beta}$, be the normal connection forms defined by $\omega_{\alpha \beta}(X)=$ $\left\langle\nabla \frac{1}{X} e_{\alpha}, e_{\beta}\right\rangle$. Then we have

$$
\begin{aligned}
& \sum_{\alpha} A_{\alpha} \circ A_{\nabla_{X}^{\perp} e_{\alpha}}+\sum_{\alpha} A_{\nabla \frac{\perp}{X} e_{\alpha}} \circ A_{\alpha} \\
& =\sum_{\alpha, \beta} \omega_{\alpha \beta}(X) A_{\alpha} \circ A_{\beta}+\sum_{\alpha, \beta} \omega_{\alpha \beta}(X) A_{\beta} \circ A_{\alpha} .
\end{aligned}
$$

On the other hand, it is obvious that $\omega_{\alpha \beta}=-\omega_{\beta \alpha}$. Hence we finally get

$$
\begin{aligned}
& \sum_{\alpha} A_{\alpha} \circ A_{\nabla \frac{1}{X} e_{\alpha}}+\sum_{\alpha} A_{\nabla \frac{1}{X} e_{\alpha}} \circ A_{\alpha} \\
& =\sum_{\alpha, \beta} \omega_{\alpha \beta}(X) A_{\alpha} \circ A_{\beta}-\sum_{\alpha, \beta} \omega_{\alpha \beta}(X) A_{\alpha} \circ A_{\beta}=0,
\end{aligned}
$$

and this completes the proof of the lemma.

Proof of Proposition 3.1. We use the well-known relation between a metric and the corresponding Riemannian connection

$$
\begin{aligned}
2 \mathrm{III}\left(\nabla_{X}^{\mathrm{III}} Y, Z\right)= & Y(\operatorname{III}(Z, X))+X(\operatorname{III}(Z, Y))-Z(\operatorname{III}(X, Y)) \\
& -\operatorname{III}([Y, Z], X)-\operatorname{III}([X, Z], Y)-\operatorname{III}([Y, X], Z) .
\end{aligned}
$$

By virtue of (2.1) and (2.2), we have

$$
\begin{aligned}
2 \mathrm{III}\left(\nabla_{X}^{\mathrm{III}} Y, Z\right)= & \sum_{\alpha} Y\left\langle A_{\alpha} Z, A_{\alpha} X\right\rangle+\sum_{\alpha} X\left\langle A_{\alpha} Z, A_{\alpha} Y\right\rangle \\
& -\sum_{\alpha} Z\left\langle A_{\alpha} X, A_{\alpha} Y\right\rangle-\sum_{\alpha}\left\langle A_{\alpha}[Y, Z], A_{\alpha} X\right\rangle \\
& -\sum_{\alpha}\left\langle A_{\alpha}[X, Z], A_{\alpha} Y\right\rangle-\sum_{\alpha}\left\langle A_{\alpha}[Y, X], A_{\alpha} Z\right\rangle,
\end{aligned}
$$


or equivalently

$$
\begin{aligned}
2 \mathrm{III}\left(\nabla_{X}^{\mathrm{III}} Y, Z\right)= & \sum_{\alpha}\left\langle\left(\nabla_{Y} A_{\alpha}\right) Z-\left(\nabla_{Z} A_{\alpha}\right) Y, A_{\alpha} X\right\rangle \\
& +\sum_{\alpha}\left\langle\left(\nabla_{X} A_{\alpha}\right) Z-\left(\nabla_{Z} A_{\alpha}\right) X, A_{\alpha} Y\right\rangle \\
& +\sum_{\alpha}\left\langle\left(\nabla_{Y} A_{\alpha}\right) X, A_{\alpha} Z\right\rangle+\sum_{\alpha}\left\langle\left(\nabla_{X} A_{\alpha}\right) Y, A_{\alpha} Z\right\rangle \\
& +2 \sum_{\alpha}\left\langle A_{\alpha}\left(\nabla_{X} Y\right), A_{\alpha} Z\right\rangle .
\end{aligned}
$$

Using the Codazzi equation, we obtain

$$
\begin{aligned}
2 \mathrm{III}\left(\nabla_{X}^{\mathrm{III}} Y, Z\right)= & 2 \sum_{\alpha}\left\langle A_{\alpha}\left(\nabla_{X}\left(A_{\alpha} Y\right)\right), Z\right\rangle \\
& +\left\langle\sum_{\alpha} A_{\alpha} \circ A_{\nabla_{Y}^{\perp} e_{\alpha}}(X)+\sum_{\alpha} A_{\nabla_{Y}^{\perp} e_{\alpha}} \circ A_{\alpha}(X), Z\right\rangle \\
& -\left\langle\sum_{\alpha} A_{\alpha} \circ A_{\nabla_{\bar{X}}^{\perp} e_{\alpha}}(Y)-\sum_{\alpha} A_{\nabla_{\bar{X}}^{\perp} e_{\alpha}} \circ A_{\alpha}(Y), Z\right\rangle \\
& -\left\langle\sum_{\alpha} A_{\alpha} \circ A_{\nabla_{\frac{1}{Z}} e_{\alpha}}(Y)+\sum_{\alpha} A_{\nabla_{\frac{1}{Z}} e_{\alpha}} \circ A_{\alpha}(Y), X\right\rangle .
\end{aligned}
$$

Appealing to (2.1) and Lemma 3.2, we get

$$
\left\langle Q\left(\nabla_{X}^{\mathrm{III}} Y\right), Z\right\rangle=\sum_{\alpha}\left\langle A_{\alpha}\left(\nabla_{X}\left(A_{\alpha} Y\right)\right), Z\right\rangle-\sum_{\alpha}\left\langle A_{\alpha}\left(A_{\nabla_{X}^{\perp} e_{\alpha}} Y\right), Z\right\rangle .
$$

This completes the proof of the proposition.

Proposition 3.3. Let $M^{n}$ be an n-dimensional submanifold of the Euclidean space $E^{n+p}$ with positive definite third fundamental form III. Then the 
curvature tensor $R^{\mathrm{III}}$ of III satisfies

$$
\begin{aligned}
& \operatorname{III}\left(R^{\mathrm{III}}(X, Y) Y, X\right) \\
& =\sum_{\alpha}\left\langle R(X, Y) A_{\alpha} Y, A_{\alpha} X\right\rangle-\sum_{\alpha}\left\langle A_{R^{\perp}(X, Y) e_{\alpha}} Y, A_{\alpha} X\right\rangle \\
& +\sum_{\alpha}\left\langle\nabla_{Y}\left(A_{\alpha} Y\right)-A_{\nabla_{Y}^{\frac{1}{Y}} e_{\alpha}} Y, \nabla_{X}\left(A_{\alpha} X\right)\right\rangle \\
& -\sum_{\alpha}\left\langle\nabla_{X}\left(A_{\alpha} Y\right)-A_{\nabla_{X}^{\perp} e_{\alpha}} Y, \nabla_{Y}\left(A_{\alpha} X\right)\right\rangle \\
& -\sum_{\alpha}\left\langle\left(\widetilde{\nabla}_{X} A_{\nabla_{Y}^{\frac{1}{Y}} e_{\alpha}}\right) Y, A_{\alpha} X\right\rangle+\sum_{\alpha}\left\langle\left(\widetilde{\nabla}_{Y} A_{\nabla_{X}^{\frac{1}{X}} e_{\alpha}}\right) Y, A_{\alpha} X\right\rangle \\
& -\sum_{\alpha}\left\langle A_{\nabla_{\bar{Y}} e_{\alpha}}\left(\nabla_{X} Y\right), A_{\alpha} X\right\rangle+\sum_{\alpha}\left\langle A_{\nabla_{X}^{\frac{1}{X}} e_{\alpha}}\left(\nabla_{Y} Y\right), A_{\alpha} X\right\rangle \\
& -\sum_{\alpha, \beta}\left\langle A_{\alpha} \circ Q^{-1} \circ A_{\beta}\left(\nabla_{Y}\left(A_{\beta} Y\right)-A_{\nabla_{Y}^{\frac{1}{Y}} e_{\beta}} Y\right), \nabla_{X}\left(A_{\alpha} X\right)\right\rangle \\
& +\sum_{\alpha, \beta}\left\langle A_{\alpha} \circ Q^{-1} \circ A_{\beta}\left(\nabla_{X}\left(A_{\beta} Y\right)-A_{\nabla_{X}^{\perp} e_{\beta}} Y\right), \nabla_{Y}\left(A_{\alpha} X\right)\right\rangle \\
& +\sum_{\alpha, \beta}\left\langle A_{\nabla_{X}^{\frac{1}{X}} e_{\alpha}} \circ A_{\alpha} \circ Q^{-1} \circ A_{\beta}\left(\nabla_{Y}\left(A_{\beta} Y\right)-A_{\nabla_{\bar{Y}} e_{\beta}} Y\right), X\right\rangle \\
& -\sum_{\alpha, \beta}\left\langle A_{\nabla_{Y}^{\perp} e_{\alpha}} \circ A_{\alpha} \circ Q^{-1} \circ A_{\beta}\left(\nabla_{X}\left(A_{\beta} Y\right)-A_{\nabla_{X}^{\perp} e_{\beta}} Y\right), X\right\rangle,
\end{aligned}
$$

where $X, Y$ are tangent vector fields of $M^{n}$.

Proof. In view of (2.1), we see that the curvature tensor $R^{\mathrm{III}}$ satisfies

$$
\begin{aligned}
\mathrm{III}\left(R^{\mathrm{III}}(X, Y) Y, X\right)= & \left\langle Q\left(\nabla_{X}^{\mathrm{III}} \nabla_{Y}^{\mathrm{III}} Y\right), X\right\rangle-\left\langle Q\left(\nabla_{Y}^{\mathrm{III}} \nabla_{X}^{\mathrm{III}} Y\right), X\right\rangle \\
& -\left\langle Q\left(\nabla_{[X, Y]}^{\mathrm{III}} Y\right), X\right\rangle .
\end{aligned}
$$

Using Proposition 3.1, we get

$$
\begin{aligned}
\mathrm{III} & \left(R^{\mathrm{III}}(X, Y) Y, X\right) \\
= & \sum_{\alpha}\left\langle\nabla_{X}\left(A_{\alpha}\left(\nabla_{Y}^{\mathrm{III}} Y\right)\right), A_{\alpha} X\right\rangle-\sum_{\alpha}\left\langle\nabla_{Y}\left(A_{\alpha}\left(\nabla_{X}^{\mathrm{III}} Y\right)\right), A_{\alpha} X\right\rangle \\
& -\sum_{\alpha}\left\langle A_{\alpha}\left(A_{\nabla_{X}^{\perp} e_{\alpha}}\left(\nabla_{Y}^{\mathrm{III}} Y\right)\right), X\right\rangle+\sum_{\alpha}\left\langle A_{\alpha}\left(A_{\nabla_{Y}^{\perp} e_{\alpha}}\left(\nabla_{X}^{\mathrm{III}} Y\right)\right), X\right\rangle \\
& -\sum_{\alpha}\left\langle\nabla_{[X, Y]}\left(A_{\alpha} Y\right), A_{\alpha} X\right\rangle+\sum_{\alpha}\left\langle A_{\alpha}\left(A_{\nabla_{[X, Y]}^{\perp} e_{\alpha}} Y\right), X\right\rangle,
\end{aligned}
$$


or equivalently

$$
\begin{aligned}
\operatorname{III} & \left(R^{\mathrm{III}}(X, Y) Y, X\right) \\
= & \sum_{\alpha} X\left\langle A_{\alpha}\left(\nabla_{Y}^{\mathrm{III}} Y\right), A_{\alpha} X\right\rangle-\sum_{\alpha} Y\left\langle A_{\alpha}\left(\nabla_{X}^{\mathrm{III}} Y\right), A_{\alpha} X\right\rangle \\
& -\sum_{\alpha}\left\langle A_{\alpha}\left(\nabla_{Y}^{\mathrm{III}} Y\right), \nabla_{X}\left(A_{\alpha} X\right)\right\rangle+\sum_{\alpha}\left\langle A_{\alpha}\left(\nabla_{X}^{\mathrm{III}} Y\right), \nabla_{Y}\left(A_{\alpha} X\right)\right\rangle \\
& -\sum_{\alpha}\left\langle A_{\alpha}\left(A_{\nabla_{X}^{\perp} e_{\alpha}}\left(\nabla_{Y}^{\mathrm{III}} Y\right)\right), X\right\rangle+\sum_{\alpha}\left\langle A_{\alpha}\left(A_{\nabla_{Y}^{\perp} e_{\alpha}}\left(\nabla_{X}^{\mathrm{III}} Y\right)\right), X\right\rangle \\
& -\sum_{\alpha}\left\langle\nabla_{[X, Y]}\left(A_{\alpha} Y\right), A_{\alpha} X\right\rangle+\sum_{\alpha}\left\langle A_{\alpha}\left(A_{\nabla_{[X, Y]}^{\perp} e_{\alpha}} Y\right), X\right\rangle .
\end{aligned}
$$

Bearing in mind (2.2), we have

$$
\begin{aligned}
\mathrm{III} & \left(R^{\mathrm{III}}(X, Y) Y, X\right) \\
= & X\left\langle Q\left(\nabla_{Y}^{\mathrm{III}} Y\right), X\right\rangle-Y\left\langle Q\left(\nabla_{X}^{\mathrm{III}} Y\right), X\right\rangle \\
& -\sum_{\alpha}\left\langle A_{\alpha}\left(\nabla_{Y}^{\mathrm{III}} Y\right), \nabla_{X}\left(A_{\alpha} X\right)\right\rangle+\sum_{\alpha}\left\langle A_{\alpha}\left(\nabla_{X}^{\mathrm{III}} Y\right), \nabla_{Y}\left(A_{\alpha} X\right)\right\rangle \\
& -\sum_{\alpha}\left\langle A_{\alpha}\left(A_{\nabla_{X}^{\perp} e_{\alpha}}\left(\nabla_{Y}^{\mathrm{III}} Y\right)\right), X\right\rangle+\sum_{\alpha}\left\langle A_{\alpha}\left(A_{\nabla_{Y}^{\perp} e_{\alpha}}\left(\nabla_{X}^{\mathrm{III}} Y\right)\right), X\right\rangle \\
& -\sum_{\alpha}\left\langle\nabla_{[X, Y]}\left(A_{\alpha} Y\right), A_{\alpha} X\right\rangle+\sum_{\alpha}\left\langle A_{\alpha}\left(A_{\nabla_{[X, Y]}^{\perp} e_{\alpha}} Y\right), X\right\rangle .
\end{aligned}
$$

Now using Proposition 3.1, we obtain

$$
\begin{aligned}
\operatorname{III} & \left(R^{\mathrm{III}}(X, Y) Y, X\right) \\
= & \sum_{\alpha} X\left\langle\nabla_{Y}\left(A_{\alpha} Y\right), A_{\alpha} X\right\rangle-\sum_{\alpha} Y\left\langle\nabla_{X}\left(A_{\alpha} Y\right), A_{\alpha} X\right\rangle \\
& -\sum_{\alpha} X\left\langle A_{\nabla_{Y}^{\perp} e_{\alpha}} Y, A_{\alpha} X\right\rangle+\sum_{\alpha} Y\left\langle A_{\nabla_{X}^{\perp} e_{\alpha}} Y, A_{\alpha} X\right\rangle \\
& -\sum_{\alpha}\left\langle\nabla_{[X, Y]}\left(A_{\alpha} Y\right), A_{\alpha} X\right\rangle+\sum_{\alpha}\left\langle A_{\alpha}\left(A_{\nabla_{[X, Y]}^{\perp}} e_{\alpha} Y\right), X\right\rangle \\
& -\sum_{\alpha}\left\langle A_{\alpha}\left(\nabla_{Y}^{\mathrm{III}} Y\right), \nabla_{X}\left(A_{\alpha} X\right)\right\rangle+\sum_{\alpha}\left\langle A_{\alpha}\left(\nabla_{X}^{\mathrm{III}} Y\right), \nabla_{Y}\left(A_{\alpha} X\right)\right\rangle \\
& -\sum_{\alpha}\left\langle A_{\alpha}\left(A_{\nabla_{X}^{\perp} e_{\alpha}}\left(\nabla_{Y}^{\mathrm{III}} Y\right)\right), X\right\rangle+\sum_{\alpha}\left\langle A_{\alpha}\left(A_{\nabla_{Y}^{\perp} e_{\alpha}}\left(\nabla_{X}^{\mathrm{III}} Y\right)\right), X\right\rangle,
\end{aligned}
$$


or equivalently

$$
\begin{aligned}
\mathrm{III} & \left.R^{\mathrm{III}}(X, Y) Y, X\right) \\
= & \sum_{\alpha}\left\langle R(X, Y) A_{\alpha} Y, A_{\alpha} X\right\rangle-\sum_{\alpha}\left\langle\nabla_{X}\left(A_{\alpha} Y\right), \nabla_{Y}\left(A_{\alpha} X\right)\right\rangle \\
& +\sum_{\alpha}\left\langle\nabla_{Y}\left(A_{\alpha} Y\right), \nabla_{X}\left(A_{\alpha} X\right)\right\rangle \\
& +\sum_{\alpha}\left\langle A_{\nabla_{X}^{\perp} e_{\alpha}} Y, \nabla_{Y}\left(A_{\alpha} X\right)\right\rangle-\sum_{\alpha}\left\langle A_{\nabla_{Y}^{\perp} e_{\alpha}} Y, \nabla_{X}\left(A_{\alpha} X\right)\right\rangle \\
& -\sum_{\alpha}\left\langle\nabla_{X}\left(A_{\nabla_{Y}^{\frac{1}{Y}} e_{\alpha}} Y\right), A_{\alpha} X\right\rangle+\sum_{\alpha}\left\langle\nabla_{Y}\left(A_{\nabla_{X}^{\perp} e_{\alpha}} Y\right), A_{\alpha} X\right\rangle \\
& +\sum_{\alpha}\left\langle A_{\nabla_{[X, Y]}^{\perp}} e_{\alpha} Y, A_{\alpha} X\right\rangle \\
& -\sum_{\alpha}\left\langle A_{\alpha}\left(\nabla_{Y}^{\mathrm{III}} Y\right), \nabla_{X}\left(A_{\alpha} X\right)\right\rangle+\sum_{\alpha}\left\langle A_{\alpha}\left(\nabla_{X}^{\mathrm{III}} Y\right), \nabla_{Y}\left(A_{\alpha} X\right)\right\rangle \\
& -\sum_{\alpha}\left\langle A_{\alpha} \circ A_{\nabla_{X}^{\frac{1}{X}} e_{\alpha}}\left(\nabla_{Y}^{\mathrm{III}} Y\right), X\right\rangle+\sum_{\alpha}\left\langle A_{\alpha} \circ A_{\nabla_{Y}^{\perp} e_{\alpha}}\left(\nabla_{X}^{\mathrm{III}} Y\right), X\right\rangle .
\end{aligned}
$$

Appealing again to Proposition 3.1, we take

$$
\begin{aligned}
& \mathrm{III}\left(R^{\mathrm{III}}(X, Y) Y, X\right) \\
& =\sum_{\alpha}\left\langle R(X, Y) A_{\alpha} Y, A_{\alpha} X\right\rangle-\sum_{\alpha}\left\langle\nabla_{X}\left(A_{\alpha} Y\right), \nabla_{Y}\left(A_{\alpha} X\right)\right\rangle \\
& +\sum_{\alpha}\left\langle\nabla_{Y}\left(A_{\alpha} Y\right), \nabla_{X}\left(A_{\alpha} X\right)\right\rangle \\
& +\sum_{\alpha}\left\langle A_{\nabla_{X}^{\frac{1}{X}} e_{\alpha}} Y, \nabla_{Y}\left(A_{\alpha} X\right)\right\rangle-\sum_{\alpha}\left\langle A_{\nabla_{Y}^{\frac{1}{Y}} e_{\alpha}} Y, \nabla_{X}\left(A_{\alpha} X\right)\right\rangle \\
& -\sum_{\alpha}\left\langle\nabla_{X}\left(A_{\nabla_{Y}^{\perp} e_{\alpha}} Y\right), A_{\alpha} X\right\rangle+\sum_{\alpha}\left\langle\nabla_{Y}\left(A_{\nabla_{X}^{\perp} e_{\alpha}} Y\right), A_{\alpha} X\right\rangle \\
& +\sum_{\alpha}\left\langle A_{\nabla_{[X, Y]}^{\perp} e_{\alpha}} Y, A_{\alpha} X\right\rangle \\
& -\sum_{\alpha, \beta}\left\langle A_{\alpha} \circ Q^{-1} \circ A_{\beta}\left(\nabla_{Y}\left(A_{\beta} Y\right)-A_{\nabla_{Y}^{\perp} e_{\beta}} Y\right), \nabla_{X}\left(A_{\alpha} X\right)\right\rangle \\
& +\sum_{\alpha, \beta}\left\langle A_{\alpha} \circ Q^{-1} \circ A_{\beta}\left(\nabla_{X}\left(A_{\beta} Y\right)-A_{\nabla_{X}^{\perp} e_{\beta}} Y\right), \nabla_{Y}\left(A_{\alpha} X\right)\right\rangle \\
& -\sum_{\alpha, \beta}\left\langle A_{\alpha} \circ A_{\nabla_{X}^{\perp} e_{\alpha}} \circ Q^{-1} \circ A_{\beta}\left(\nabla_{Y}\left(A_{\beta} Y\right)-A_{\nabla_{Y}^{\perp} e_{\beta}} Y\right), X\right\rangle \\
& +\sum_{\alpha, \beta}\left\langle A_{\alpha} \circ A_{\nabla_{\bar{Y}} e_{\alpha}} \circ Q^{-1} \circ A_{\beta}\left(\nabla_{X}\left(A_{\beta} Y\right)-A_{\nabla_{\bar{X}} e_{\beta}} Y\right), X\right\rangle,
\end{aligned}
$$


or equivalently

$$
\begin{aligned}
& \operatorname{III}\left(R^{\mathrm{III}}(X, Y) Y, X\right) \\
& =\sum_{\alpha}\left\langle R(X, Y) A_{\alpha} Y, A_{\alpha} X\right\rangle \\
& +\sum_{\alpha}\left\langle\nabla_{Y}\left(A_{\alpha} Y\right)-A_{\nabla_{Y}^{\frac{1}{Y}} e_{\alpha}} Y, \nabla_{X}\left(A_{\alpha} X\right)\right\rangle \\
& -\sum_{\alpha}\left\langle\nabla_{X}\left(A_{\alpha} Y\right)-A_{\nabla_{X}^{\frac{1}{X}} e_{\alpha}} Y, \nabla_{Y}\left(A_{\alpha} X\right)\right\rangle \\
& +\sum_{\alpha}\left\langle A_{\nabla_{X}^{\frac{1}{X}} e_{\alpha}}\left(\nabla_{Y} Y\right), A_{\alpha} X\right\rangle-\sum_{\alpha}\left\langle A_{\nabla_{\bar{Y}}} e_{\alpha}\left(\nabla_{X} Y\right), A_{\alpha} X\right\rangle \\
& -\sum_{\alpha}\left\langle\left(\widetilde{\nabla}_{X} A_{\nabla_{Y}^{\perp} e_{\alpha}}\right) Y, A_{\alpha} X\right\rangle+\sum_{\alpha}\left\langle\left(\widetilde{\nabla}_{Y} A_{\nabla_{X}^{\perp} e_{\alpha}}\right) Y, A_{\alpha} X\right\rangle \\
& -\sum_{\alpha}\left\langle A_{\nabla_{X}^{\perp} \nabla_{Y}^{\frac{1}{Y}} e_{\alpha}} Y, A_{\alpha} X\right\rangle+\sum_{\alpha}\left\langle A_{\nabla_{Y}} \nabla_{\frac{1}{X}}^{\perp} e_{\alpha} Y, A_{\alpha} X\right\rangle \\
& +\sum_{\alpha}\left\langle A_{\nabla_{[X, Y]}^{\perp} e_{\alpha}} Y, A_{\alpha} X\right\rangle \\
& -\sum_{\alpha, \beta}\left\langle A_{\alpha} \circ Q^{-1} \circ A_{\beta}\left(\nabla_{Y}\left(A_{\beta} Y\right)-A_{\nabla_{\bar{Y}}^{\perp} e_{\beta}} Y\right), \nabla_{X}\left(A_{\alpha} X\right)\right\rangle \\
& +\sum_{\alpha, \beta}\left\langle A_{\alpha} \circ Q^{-1} \circ A_{\beta}\left(\nabla_{X}\left(A_{\beta} Y\right)-A_{\nabla_{X}^{\frac{1}{X}} e_{\beta}} Y\right), \nabla_{Y}\left(A_{\alpha} X\right)\right\rangle \\
& -\sum_{\alpha, \beta}\left\langle A_{\alpha} \circ A_{\nabla_{X}^{\perp} e_{\alpha}} \circ Q^{-1} \circ A_{\beta}\left(\nabla_{Y}\left(A_{\beta} Y\right)-A_{\nabla_{Y}^{\perp} e_{\beta}} Y\right), X\right\rangle \\
& +\sum_{\alpha, \beta}\left\langle A_{\alpha} \circ A_{\nabla_{Y}^{\perp} e_{\alpha}} \circ Q^{-1} \circ A_{\beta}\left(\nabla_{X}\left(A_{\beta} Y\right)-A_{\nabla_{X}^{\perp} e_{\beta}} Y\right), X\right\rangle .
\end{aligned}
$$

Now bearing in mind the definition of the normal curvature tensor, and using Lemma 3.2, we obtain the desired relation.

\section{The third fundamental form of minimal submanifolds.}

Lemma 4.1. Let $M^{n}$ be an n-dimensional minimal submanifold of the Euclidean space $E^{n+p}$ with negative Ricci curvature. Let $x \in M^{n},\left\{e_{1}, \ldots, e_{n}\right\}$ be an orthonormal basis of $T_{x} M^{n}$, and extend them to orthonormal vector fields $E_{1}, \ldots, E_{n}$ in a neighborhood of $x$ such that $\nabla E_{i}=0$ at $x$. Then we 
have at $x$

$$
\begin{aligned}
& \sum_{i, j} \operatorname{III}\left(R^{\mathrm{III}}\left(e_{i}, e_{j}\right) e_{j}, e_{i}\right) \\
& =\sum_{i, j, \alpha}\left\langle R\left(e_{i}, e_{j}\right) A_{\alpha} e_{j}, A_{\alpha} e_{i}\right\rangle \\
& \quad-\sum_{i, j, \alpha}\left\langle A_{\left.R^{\perp}\left(E_{i}, E_{j}\right) e_{\alpha} E_{j}, A_{\alpha} E_{i}\right\rangle-\|\widetilde{\nabla} B\|^{2}}\right. \\
& \quad+\sum_{i, j, \alpha, \beta}\left\langle A_{\alpha} \circ Q^{-1} \circ A_{\beta}\left(\left(\widetilde{\nabla}_{E_{i}} A_{\beta}\right) E_{j}\right),\left(\widetilde{\nabla}_{E_{i}} A_{\alpha}\right) E_{j}\right\rangle
\end{aligned}
$$

where $\|\widetilde{\nabla} B\|^{2}$ is given by

$$
\|\widetilde{\nabla} B\|^{2}=\sum_{i, j, \alpha}\left\|\left(\widetilde{\nabla}_{E_{i}} A_{\alpha}\right) E_{j}\right\|^{2} .
$$

Proof. Using Proposition 3.3, and bearing in mind the fact that $\nabla E_{i}=0$ at $x$, we get at $x$

$$
\begin{aligned}
\sum_{i, j} & \operatorname{III}\left(R^{\mathrm{III}}\left(e_{i}, e_{j}\right) e_{j}, e_{i}\right) \\
= & \sum_{i, j, \alpha}\left\langle R\left(e_{i}, e_{j}\right) A_{\alpha} e_{j}, A_{\alpha} e_{i}\right\rangle \\
& +\sum_{i, j, \alpha}\left\langle\left(\widetilde{\nabla}_{E_{j}} A_{\alpha}\right) E_{j}, \nabla_{E_{i}}\left(A_{\alpha} E_{i}\right)\right\rangle-\sum_{i, j, \alpha}\left\langle\left(\widetilde{\nabla}_{E_{i}} A_{\alpha}\right) E_{j}, \nabla_{E_{j}}\left(A_{\alpha} E_{i}\right)\right\rangle \\
& -\sum_{i, j, \alpha}\left\langle\left(\widetilde{\nabla}_{E_{i}} A_{\nabla_{E_{j}} e_{\alpha}}\right) E_{j}, A_{\alpha} E_{i}\right\rangle+\sum_{i, j, \alpha}\left\langle\left(\widetilde{\nabla}_{E_{j}} A_{\nabla_{E_{i}}^{\perp} e_{\alpha}}\right) E_{j}, A_{\alpha} E_{i}\right\rangle \\
& -\sum_{i, j, \alpha}\left\langle A_{R^{\perp}\left(E_{i}, E_{j}\right) e_{\alpha}} E_{j}, A_{\alpha} E_{i}\right\rangle \\
& -\sum_{i, j, \alpha, \beta}\left\langle A_{\alpha} \circ Q^{-1} \circ A_{\beta}\left(\left(\widetilde{\nabla}_{E_{j}} A_{\beta}\right) E_{j}\right), \nabla_{E_{i}}\left(A_{\alpha} E_{i}\right)\right\rangle \\
& +\sum_{i, j, \alpha, \beta}\left\langle A_{\alpha} \circ Q^{-1} \circ A_{\beta}\left(\left(\widetilde{\nabla}_{E_{i}} A_{\beta}\right) E_{j}\right), \nabla_{E_{j}}\left(A_{\alpha} E_{i}\right)\right\rangle \\
& +\sum_{i, j, \alpha, \beta}\left\langle A_{\nabla_{E_{i}}^{\perp} e_{\alpha}} \circ A_{\alpha} \circ Q^{-1} \circ A_{\beta}\left(\left(\widetilde{\nabla}_{E_{j}} A_{\beta}\right) E_{j}\right), E_{i}\right\rangle \\
& -\sum_{i, j, \alpha, \beta}\left\langle A_{\nabla_{E_{j}}^{\perp} e_{\alpha}} \circ A_{\alpha} \circ Q^{-1} \circ A_{\beta}\left(\left(\widetilde{\nabla}_{E_{i}} A_{\beta}\right) E_{j}\right), E_{i}\right\rangle .
\end{aligned}
$$


However, the fact that $M^{n}$ is minimal ensures that

$$
\sum_{i}\left(\widetilde{\nabla}_{E_{i}} A_{\xi}\right) E_{i}=0
$$

where $\xi$ is any normal vector field. Hence we take

$$
\begin{aligned}
& \sum_{i, j} \operatorname{III}\left(R^{\mathrm{III}}\left(e_{i}, e_{j}\right) e_{j}, e_{i}\right) \\
& =\sum_{i, j, \alpha}\left\langle R\left(e_{i}, e_{j}\right) A_{\alpha} e_{j}, A_{\alpha} e_{i}\right\rangle \\
& \quad-\sum_{i, j, \alpha}\left\langle\left(\widetilde{\nabla}_{E_{i}} A_{\alpha}\right) E_{j}, \nabla_{E_{j}}\left(A_{\alpha} E_{i}\right)\right\rangle-\sum_{i, j, \alpha}\left\langle\left(\widetilde{\nabla}_{E_{i}} A_{\nabla_{E_{j}}} e_{\alpha}\right) E_{j}, A_{\alpha} E_{i}\right\rangle \\
& \quad-\sum_{i, j, \alpha}\left\langle A_{R^{\perp}\left(E_{i}, E_{j}\right) e_{\alpha}} E_{j}, A_{\alpha} E_{i}\right\rangle \\
& \quad+\sum_{i, j, \alpha, \beta}\left\langle A_{\alpha} \circ Q^{-1} \circ A_{\beta}\left(\left(\widetilde{\nabla}_{E_{i}} A_{\beta}\right) E_{j}\right),\left(\widetilde{\nabla}_{E_{j}} A_{\alpha}\right) E_{i}\right\rangle .
\end{aligned}
$$

On the other hand, it is easy to verify that

$$
\sum_{i, j, \alpha}\left\langle\left(\widetilde{\nabla}_{E_{i}} A_{\nabla_{E_{j}}^{\perp} e_{\alpha}}\right) E_{j}, A_{\alpha} E_{i}\right\rangle=-\sum_{i, j, \alpha}\left\langle\left(\widetilde{\nabla}_{E_{i}} A_{\alpha}\right) E_{j}, A_{\nabla_{E_{j}}^{\perp} e_{\alpha}} E_{i}\right\rangle .
$$

Therefore, we obtain

$$
\begin{aligned}
& \sum_{i, j} \operatorname{III}\left(R^{\mathrm{III}}\left(e_{i}, e_{j}\right) e_{j}, e_{i}\right) \\
& =\sum_{i, j, \alpha}\left\langle R\left(e_{i}, e_{j}\right) A_{\alpha} e_{j}, A_{\alpha} e_{i}\right\rangle \\
& \quad-\sum_{i, j, \alpha}\left\langle\left(\widetilde{\nabla}_{E_{i}} A_{\alpha}\right) E_{j},\left(\widetilde{\nabla}_{E_{j}} A_{\alpha}\right) E_{i}\right\rangle-\sum_{i, j, \alpha}\left\langle A_{R^{\perp}\left(E_{i}, E_{j}\right) e_{\alpha}} E_{j}, A_{\alpha} E_{i}\right\rangle \\
& \quad+\sum_{i, j, \alpha, \beta}\left\langle A_{\alpha} \circ Q^{-1} \circ A_{\beta}\left(\left(\widetilde{\nabla}_{E_{i}} A_{\beta}\right) E_{j}\right),\left(\widetilde{\nabla}_{E_{j}} A_{\alpha}\right) E_{i}\right\rangle,
\end{aligned}
$$

and appealing to the Codazzi equation, we get the desired relation.

For each matrix $A$ we denote by $N(A)$ the square of the norm of $A$, i.e.,

$$
N(A)=\operatorname{tr}\left(A A^{t}\right) .
$$

Lemma 4.2. Let $M^{n}$ be as in Lemma 4.1, and let $H_{\alpha}=\left(h_{i j}^{\alpha}\right)$ denote the symmetric matrix of the shape operator $A_{\alpha}$ with respect to the orthonormal 
basis $\left\{E_{1}, \ldots, E_{n}\right\}$ for each $\alpha$. Then we have

$$
\begin{aligned}
& \inf K_{\mathrm{III}}\left(\tau^{2}-\|\mathrm{Ric}\|^{2}\right) \\
& \leq-\|\mathrm{Ric}\|^{2}-\|\widetilde{\nabla} B\|^{2} \\
& \quad+\sum_{\alpha, \beta}\left(\operatorname{tr}\left(H_{\alpha} H_{\beta}\right)\right)^{2}+\sum_{\alpha, \beta} N\left(H_{\alpha} H_{\beta}-H_{\beta} H_{\alpha}\right) \\
& \quad+\sum_{i, j, \alpha, \beta}\left\langle A_{\alpha} \circ Q^{-1} \circ A_{\beta}\left(\left(\widetilde{\nabla}_{E_{i}} A_{\beta}\right) E_{j}\right),\left(\widetilde{\nabla}_{E_{i}} A_{\alpha}\right) E_{j}\right\rangle .
\end{aligned}
$$

Proof. Let $x$ be an arbitrary point in $M^{n},\left\{e_{1}, \ldots, e_{n}\right\}$ be an orthonormal basis of $T_{x} M^{n}$, and extend them to orthonormal vector fields $E_{1}, \ldots, E_{n}$ in a neighborhood of $x$ such that $\nabla E_{i}=0$ at $x$. Then we have at $x$

$$
\begin{aligned}
& \sum_{i \neq j} \operatorname{III}\left(R^{\mathrm{III}}\left(e_{i}, e_{j}\right) e_{j}, e_{i}\right) \\
& =\sum_{i \neq j} K_{\mathrm{III}}\left(e_{i} \wedge e_{j}\right)\left(\operatorname{III}\left(e_{i}, e_{i}\right) \operatorname{III}\left(e_{j}, e_{j}\right)-\left(\operatorname{III}\left(e_{i}, e_{j}\right)\right)^{2}\right),
\end{aligned}
$$

where $K_{\mathrm{III}}\left(e_{i} \wedge e_{j}\right)$ denotes the sectional curvature of III for the 2-plane spanned by $e_{i}$ and $e_{j}$. This implies that

$$
\begin{aligned}
& \inf K_{\mathrm{III}} \sum_{i \neq j}\left(\operatorname{III}\left(e_{i}, e_{i}\right) \operatorname{III}\left(e_{j}, e_{j}\right)-\left(\operatorname{III}\left(e_{i}, e_{j}\right)\right)^{2}\right) \\
& \leq \sum_{i \neq j} \operatorname{III}\left(R^{\mathrm{III}}\left(e_{i}, e_{j}\right) e_{j}, e_{i}\right) .
\end{aligned}
$$

On the other hand, we immediately verify that

$$
\begin{aligned}
& \sum_{i \neq j}\left(\operatorname{III}\left(e_{i}, e_{i}\right) \operatorname{III}\left(e_{j}, e_{j}\right)-\left(\operatorname{III}\left(e_{i}, e_{j}\right)\right)^{2}\right) \\
& =\left(\sum_{i, \alpha}\left\langle A_{\alpha}^{2} e_{i}, e_{i}\right\rangle\right)^{2}-\sum_{i, j}\left(\sum_{\alpha}\left\langle A_{\alpha}^{2} e_{i}, e_{j}\right\rangle\right)^{2} \\
& =S^{2}-\sum_{\alpha, \beta} \operatorname{tr}\left(A_{\alpha}^{2} \circ A_{\beta}^{2}\right) .
\end{aligned}
$$

Using the Gauss equation and the fact that $M^{n}$ is minimal we find that $S=-\tau$, and taking (2.2) into account, we obtain

$$
\sum_{i \neq j}\left(\operatorname{III}\left(e_{i}, e_{i}\right) \operatorname{III}\left(e_{j}, e_{j}\right)-\left(\operatorname{III}\left(e_{i}, e_{j}\right)\right)^{2}\right)=\tau^{2}-\|\operatorname{Ric}\|^{2} .
$$

Therefore, we finally have

$$
\inf K_{\mathrm{III}}\left(\tau^{2}-\|\operatorname{Ric}\|^{2}\right) \leq \sum_{i \neq j} \operatorname{III}\left(R^{\mathrm{III}}\left(e_{i}, e_{j}\right) e_{j}, e_{i}\right) .
$$


Using Lemma 4.1, we shall compute the right hand side of (4.1). The Gauss equation yields

$$
\sum_{i, j, \alpha}\left\langle R\left(e_{i}, e_{j}\right) A_{\alpha} e_{j}, A_{\alpha} e_{i}\right\rangle=\sum_{\alpha, \beta}\left(\operatorname{tr}\left(A_{\alpha} \circ A_{\beta}\right)\right)^{2}-\sum_{\alpha, \beta} \operatorname{tr}\left(A_{\alpha} \circ A_{\beta}\right)^{2} .
$$

Appealing to the Ricci equation, we get

$$
\begin{aligned}
& \sum_{i, j, \alpha}\left\langle A_{R^{\perp}\left(E_{i}, E_{j}\right) e_{\alpha}} E_{j}, A_{\alpha} E_{i}\right\rangle \\
& =\sum_{i, j, \alpha, \beta}\left(\left\langle A_{\alpha} e_{i}, A_{\beta} e_{j}\right\rangle\left\langle A_{\alpha} e_{j}, A_{\beta} e_{i}\right\rangle-\left\langle A_{\alpha} e_{i}, A_{\beta} e_{j}\right\rangle^{2}\right),
\end{aligned}
$$

or equivalently

$$
\sum_{i, j, \alpha}\left\langle A_{R^{\perp}\left(E_{i}, E_{j}\right) e_{\alpha}} E_{j}, A_{\alpha} E_{i}\right\rangle=\sum_{\alpha, \beta} \operatorname{tr}\left(A_{\alpha} \circ A_{\beta}\right)^{2}-\sum_{\alpha, \beta} \operatorname{tr}\left(A_{\alpha}^{2} \circ A_{\beta}^{2}\right) .
$$

By virtue of Lemma 4.1, (4.2) and (4.3), (4.1) yields

$$
\begin{aligned}
& \inf K_{\mathrm{III}}\left(\tau^{2}-\|\operatorname{Ric}\|^{2}\right) \\
& \leq-\|\operatorname{Ric}\|^{2}+\sum_{\alpha, \beta}\left(\operatorname{tr}\left(A_{\alpha} \circ A_{\beta}\right)\right)^{2}-\|\widetilde{\nabla} B\|^{2} \\
& \quad+2 \sum_{\alpha, \beta} \operatorname{tr}\left(A_{\alpha}^{2} \circ A_{\beta}^{2}\right)-2 \sum_{\alpha, \beta} \operatorname{tr}\left(A_{\alpha} \circ A_{\beta}\right)^{2} \\
& \quad+\sum_{i, j, \alpha, \beta}\left\langle A_{\alpha} \circ Q^{-1} \circ A_{\beta}\left(\left(\widetilde{\nabla}_{E_{i}} A_{\beta}\right) E_{j}\right),\left(\widetilde{\nabla}_{E_{i}} A_{\alpha}\right) E_{j}\right\rangle,
\end{aligned}
$$

from which we immediately deduce the desired inequality.

\section{Proofs of the results.}

We need the following result which was proved in $[\mathbf{1 3}]$.

Lemma 5.1. Let $H_{1}, \ldots, H_{p}$ be symmetric $(n \times n)$-matrices. Then

$$
\sum_{\alpha, \beta=1}^{p}\left(\operatorname{tr}\left(H_{\alpha} H_{\beta}\right)\right)^{2}+\sum_{\alpha, \beta=1}^{p} N\left(H_{\alpha} H_{\beta}-H_{\beta} H_{\alpha}\right) \leq \frac{3}{2}\left(\sum_{\alpha=1}^{p} N\left(H_{\alpha}\right)\right)^{2},
$$

and the equality holds if and only if one of the following conditions holds:

1) $H_{1}=\cdots=H_{p}=0$,

2) only two of the matrices $H_{1}, \ldots, H_{p}$ are different from zero. Moreover, assuming $H_{1} \neq 0, H_{2} \neq 0, H_{3}=\cdots=H_{p}=0$, then $N\left(H_{1}\right)=N\left(H_{2}\right)$, 
and there exists an orthogonal $(n \times n)$-matrix $T$ such that

$$
T H_{1} T^{t}=\sqrt{\frac{N\left(H_{1}\right)}{2}}\left(\begin{array}{ccccc}
1 & 0 & 0 & \ldots & 0 \\
0 & -1 & 0 & \ldots & 0 \\
0 & 0 & 0 & \ldots & 0 \\
& & . & & \\
& & . & & \\
& & . & & \\
0 & 0 & 0 & \ldots & 0
\end{array}\right)
$$

and

$$
T H_{2} T^{t}=\sqrt{\frac{N\left(H_{1}\right)}{2}}\left(\begin{array}{ccccc}
0 & 1 & 0 & \ldots & 0 \\
1 & 0 & 0 & \ldots & 0 \\
0 & 0 & 0 & \ldots & 0 \\
& & . & & \\
& & . & & \\
& & . & & \\
0 & 0 & 0 & \ldots & 0
\end{array}\right)
$$

Proof of Theorem A. Let $x$ be an arbitrary point in $M^{n}$. Without loss of generality, we may choose the orthonormal basis $\left\{e_{1}, \ldots, e_{n}\right\}$ of $T_{x} M^{n}$ such that

$$
Q e_{i}=\mu_{i} e_{i},
$$

for any $i$. Moreover, we extend the basis $\left\{e_{1}, \ldots, e_{n}\right\}$ to orthonormal vector fields $E_{1}, \ldots, E_{n}$ in a neighborhood of $x$ such that $\nabla E_{i}=0$ at $x$. Then we have at $x$

$$
\mu_{i}=\sum_{j, \alpha}\left(h_{i j}^{\alpha}\right)^{2}
$$

For convenience we set

$$
h_{i j k}^{\alpha}=\left\langle\left(\widetilde{\nabla}_{E_{i}} A_{\alpha}\right) E_{j}, E_{k}\right\rangle .
$$

Moreover, we get at $x$

$$
\begin{aligned}
& \sum_{i, j, \alpha, \beta}\left\langle A_{\alpha} \circ Q^{-1} \circ A_{\beta}\left(\left(\widetilde{\nabla}_{E_{i}} A_{\beta}\right) E_{j}\right),\left(\widetilde{\nabla}_{E_{i}} A_{\alpha}\right) E_{j}\right\rangle \\
& =\sum_{i, j, k, r, m, s, \alpha, \beta} h_{i j k}^{\alpha} h_{i j m}^{\beta} h_{k r}^{\alpha} h_{m s}^{\beta}\left\langle Q^{-1} e_{r}, e_{s}\right\rangle \\
& =\sum_{i, j, k} \frac{1}{\mu_{k}}\left(\sum_{l, \alpha} h_{i j l}^{\alpha} h_{k l}^{\alpha}\right)^{2} .
\end{aligned}
$$


Using the Cauchy-Schwarz inequality, we obtain

$$
\begin{aligned}
& \sum_{i, j, \alpha, \beta}\left\langle A_{\alpha} \circ Q^{-1} \circ A_{\beta}\left(\left(\widetilde{\nabla}_{E_{i}} A_{\beta}\right) E_{j}\right),\left(\widetilde{\nabla}_{E_{i}} A_{\alpha}\right) E_{j}\right\rangle \\
& \leq \sum_{i, j, k} \frac{1}{\mu_{k}}\left(\sum_{l, \alpha}\left(h_{i j l}^{\alpha}\right)^{2}\right)\left(\sum_{l, \alpha}\left(h_{k l}^{\alpha}\right)^{2}\right) .
\end{aligned}
$$

In view of (5.1), we deduce that

$$
\sum_{i, j, \alpha, \beta}\left\langle A_{\alpha} \circ Q^{-1} \circ A_{\beta}\left(\left(\widetilde{\nabla}_{E_{i}} A_{\beta}\right) E_{j}\right),\left(\widetilde{\nabla}_{E_{i}} A_{\alpha}\right) E_{j}\right\rangle \leq n\|\widetilde{\nabla} B\|^{2},
$$

and bearing in mind Lemma 4.2, we finally get

$$
\begin{aligned}
\inf K_{\text {III }}\left(\tau^{2}-\| \text { Ric } \|^{2}\right) \leq & -\|\operatorname{Ric}\|^{2}+(n-1)\|\widetilde{\nabla} B\|^{2} \\
& +\sum_{\alpha, \beta}\left(\operatorname{tr}\left(H_{\alpha} H_{\beta}\right)\right)^{2}+\sum_{\alpha, \beta} N\left(H_{\alpha} H_{\beta}-H_{\beta} H_{\alpha}\right) .
\end{aligned}
$$

At this point we recall the well-known formula [9] for the Laplacian of the squared length $S$ of the second fundamental form of minimal submanifolds in space forms which states that

$$
\frac{1}{2} \Delta S=\|\widetilde{\nabla} B\|^{2}-\sum_{\alpha, \beta}\left(\operatorname{tr}\left(H_{\alpha} H_{\beta}\right)\right)^{2}-\sum_{\alpha, \beta} N\left(H_{\alpha} H_{\beta}-H_{\beta} H_{\alpha}\right) .
$$

Then (5.2) is written

$$
\begin{aligned}
\inf K_{\mathrm{III}}\left(\tau^{2}-\|\operatorname{Ric}\|^{2}\right) \leq & -\|\operatorname{Ric}\|^{2}+\frac{1}{2}(n-1) \Delta S \\
& +n \sum_{\alpha, \beta}\left(\operatorname{tr}\left(H_{\alpha} H_{\beta}\right)\right)^{2}+n \sum_{\alpha, \beta} N\left(H_{\alpha} H_{\beta}-H_{\beta} H_{\alpha}\right) .
\end{aligned}
$$

From the Gauss equation and the fact that $M^{n}$ is minimal, we get $S=-\tau$. Appealing to Lemma 5.1 and bearing in mind the hypothesis that the Ricci curvature is negative, we deduce that

$$
\sum_{\alpha, \beta}\left(\operatorname{tr}\left(H_{\alpha} H_{\beta}\right)\right)^{2}+\sum_{\alpha, \beta} N\left(H_{\alpha} H_{\beta}-H_{\beta} H_{\alpha}\right)<\frac{3}{2} \tau^{2} .
$$

Then (5.3) yields the desired inequality and this completes the Proof of Theorem A.

Proof of Theorem B. Let $x$ be an arbitrary point in $M^{n}$. Because of the flatness of the normal bundle, we may choose a local orthonormal frame field $\left\{e_{n+1}, \ldots, e_{n+p}\right\}$ in the normal bundle, such that $\nabla^{\perp} e_{\alpha}=0$, for any $\alpha$ (cf. [6]). Moreover, we choose an orthonormal basis $\left\{e_{1}, \ldots, e_{n}\right\}$ of $T_{x} M^{n}$ which simultaneously diagonalizes all shape operators at $x$, i.e., $h_{i j}^{\alpha}=\lambda_{i}^{\alpha} \delta_{i j}$, 
where $\lambda_{1}^{\alpha}, \ldots, \lambda_{n}^{\alpha}$ are the eigenvalues of $A_{\alpha}$, and extend them to orthonormal vector fields $E_{1}, \ldots, E_{n}$ in a neighborhood of $x$ such that $\nabla E_{i}=0$ at $x$. Then from Lemma 4.2 we have

$$
\begin{aligned}
& \inf K_{\mathrm{III}}\left(\tau^{2}-\|\operatorname{Ric}\|^{2}\right) \\
& \leq-\|\operatorname{Ric}\|^{2}-\|\widetilde{\nabla} B\|^{2}+\sum_{\alpha, \beta}\left(\operatorname{tr}\left(H_{\alpha} H_{\beta}\right)\right)^{2} \\
& \quad+\sum_{i, j, \alpha, \beta}\left\langle A_{\alpha} \circ Q^{-1} \circ A_{\beta}\left(\left(\widetilde{\nabla}_{E_{i}} A_{\beta}\right) E_{j}\right),\left(\widetilde{\nabla}_{E_{i}} A_{\alpha}\right) E_{j}\right\rangle .
\end{aligned}
$$

On the other hand, using (2.2), we easily see that at $x$ we have

$$
Q e_{i}=\mu_{i} e_{i}
$$

where $\mu_{i}$ is given by

$$
\mu_{i}=\sum_{\alpha}\left(\lambda_{i}^{\alpha}\right)^{2}
$$

Moreover, setting

$$
h_{i j k}^{\alpha}=\left\langle\left(\widetilde{\nabla}_{E_{i}} A_{\alpha}\right) E_{j}, E_{k}\right\rangle
$$

we have at $x$

$$
\begin{aligned}
& \sum_{i, j, \alpha, \beta}\left\langle A_{\alpha} \circ Q^{-1} \circ A_{\beta}\left(\left(\widetilde{\nabla}_{E_{i}} A_{\beta}\right) E_{j}\right),\left(\widetilde{\nabla}_{E_{i}} A_{\alpha}\right) E_{j}\right\rangle \\
& =\sum_{i, j, k, m, \alpha, \beta} h_{i j k}^{\alpha} h_{i j m}^{\beta} \lambda_{k}^{\alpha} \lambda_{m}^{\beta}\left\langle Q^{-1} e_{k}, e_{m}\right\rangle \\
& =\sum_{i, j, k} \frac{1}{\mu_{k}}\left(\sum_{\alpha} h_{i j k}^{\alpha} \lambda_{k}^{\alpha}\right)^{2} .
\end{aligned}
$$

Now using the Cauchy-Schwarz inequality, we obtain

$$
\begin{aligned}
& \sum_{i, j, \alpha, \beta}\left\langle A_{\alpha} \circ Q^{-1} \circ A_{\beta}\left(\left(\widetilde{\nabla}_{E_{i}} A_{\beta}\right) E_{j}\right),\left(\widetilde{\nabla}_{E_{i}} A_{\alpha}\right) E_{j}\right\rangle \\
& \leq \sum_{i, j, k} \frac{1}{\mu_{k}}\left(\sum_{\alpha}\left(h_{i j k}^{\alpha}\right)^{2}\right)\left(\sum_{\alpha}\left(\lambda_{k}^{\alpha}\right)^{2}\right),
\end{aligned}
$$

and bearing in mind (5.5), we finally get

$$
\sum_{i, j, \alpha, \beta}\left\langle A_{\alpha} \circ Q^{-1} \circ A_{\beta}\left(\left(\widetilde{\nabla}_{E_{i}} A_{\beta}\right) E_{j}\right),\left(\widetilde{\nabla}_{E_{i}} A_{\alpha}\right) E_{j}\right\rangle \leq\|\widetilde{\nabla} B\|^{2} .
$$

On the other hand, we have

$$
\sum_{\alpha, \beta}\left(\operatorname{tr}\left(H_{\alpha} H_{\beta}\right)\right)^{2}=\sum_{\alpha, \beta}\left(\sum_{i} \lambda_{i}^{\alpha} \lambda_{i}^{\beta}\right)^{2} .
$$


Using the Cauchy-Schwarz inequality, we take

$$
\sum_{\alpha, \beta}\left(\operatorname{tr}\left(H_{\alpha} H_{\beta}\right)\right)^{2} \leq \sum_{\alpha, \beta}\left(\sum_{i}\left(\lambda_{i}^{\alpha}\right)^{2}\right)\left(\sum_{i}\left(\lambda_{i}^{\beta}\right)^{2}\right),
$$

or equivalently

$$
\sum_{\alpha, \beta}\left(\operatorname{tr}\left(H_{\alpha} H_{\beta}\right)\right)^{2} \leq \tau^{2}
$$

Then from (5.4), (5.6) and (5.7), we deduce that inf $K_{\mathrm{III}} \leq 1$.

Now we assume that inf $K_{\mathrm{III}}=1$. Then inequality (5.7) becomes equality. Without loss of generality we may assume that $A_{n+1} \neq 0$. Hence we have

$$
A_{\alpha}=f_{\alpha} A_{n+1}
$$

where $f_{\alpha}$ is a function and $\alpha>n+1$. From this and the fact that each $e_{\alpha}$ is parallel in the normal bundle, we see that

$$
\left(\widetilde{\nabla}_{X} A_{\alpha}\right) Y=X\left(f_{\alpha}\right) A_{n+1} Y+f_{\alpha}\left(\widetilde{\nabla}_{X} A_{n+1}\right) Y .
$$

Then, using the Codazzi equation, we derive that

$$
X\left(f_{\alpha}\right) A_{n+1} Y=Y\left(f_{\alpha}\right) A_{n+1} X,
$$

for any tangent vector fields $X, Y$, and $\alpha>n+1$. In particular, at $x$ we have

$$
e_{i}\left(f_{\alpha}\right) \lambda_{j}^{n+1} e_{j}=e_{j}\left(f_{\alpha}\right) \lambda_{i}^{n+1} e_{i},
$$

for $i \neq j$. In view of (5.8) and the hypothesis that the Ricci curvature is negative, we infer that $\lambda_{i}^{n+1} \neq 0$ for each $i$. Then the above relation yields $e_{i}\left(f_{\alpha}\right)=0$, for any $i$, and consequently each $f_{\alpha}$ is constant. By virtue of (5.8), the second fundamental form $B$ satisfies

$$
B(X, Y)=\sum_{\alpha}\left\langle A_{\alpha} X, Y\right\rangle e_{\alpha}=\left\langle A_{n+1} X, Y\right\rangle\left(e_{n+1}+\sum_{\alpha>n+1} f_{\alpha} e_{\alpha}\right)
$$

for any tangent vector fields $X, Y$. From this we infer that the first normal space, which is spanned by the image of $B$, is one-dimensional and invariant under parallel translation with respect to the normal connection. Appealing to the well-known reduction theorem of Erbacher [11], we deduce that $M^{n}$ lies in an $(n+1)$-dimensional totally geodesic submanifold of $E^{n+p}$, and the Proof of Theorem B is complete.

\section{References}

[1] J.L. Barbosa and M. do Carmo, A necessary condition for a metric in $M^{n}$ to be minimally immersed in $R^{n+1}$, An. Acad. Brasil Cienc., 50 (1978), 451-454, MR 80e:53021, Zbl 0402.53004.

[2] Stability of minimal surfaces and eigenvalues of the Laplacian, Math. Z., 173 (1980), 13-28, MR 81h:53007, Zbl 0432.53039. 
[3] E. Calabi, Metric Riemann surfaces, in 'Contributions to the Theory of Riemann surfaces', Annals of Math. Studies, 30, Princeton University Press (1953), 77-85, MR 15,863g, Zbl 0053.05103.

[4] _ Quelques applications de l' analyse complexe aux surfaces d' aire minima, Topics in Complex Manifolds, Presses de l' Université de Montréal (1968), 58-81.

[5] M. do Carmo and M. Dajczer, Necessary and sufficient conditions for existence of minimal hypersurfaces in spaces of constant curvature, Bol. Soc. Brasil Mat., 12 (1981), 113-121, MR 84d:53061, Zbl 0604.53023.

[6] B.Y. Chen, Geometry of Submanifolds, Marcel Dekker, New York, 1973, MR 50 \#5697, Zbl 0262.53036.

[7] _ Some pinching and classification theorems for minimal submanifolds, Arch. Math., 60 (1993), 568-578, MR 94d:53093, Zbl 0811.53060.

[8] S.S. Chern, Minimal submanifolds in a Riemannian manifold, University of Kansas, Department of Mathematics Technical Report 19, University of Kansas, Lawrence, Kansas, 1968, MR 40 \#1899.

[9] S.S. Chern, M. do Carmo and S. Kobayashi, Minimal submanifolds of a sphere with second fundamental form of constant length, in 'Functional Analysis and Related Fields', 59-75, Springer-Verlag, Berlin and New York, 1970, MR 42 \#8424, Zbl 0216.44001.

[10] S.S. Chern and R. Osserman, Remarks on the Riemannian metric of a minimal submanifold, Lecture Notes in Math., 894 (1981), 49-90, MR 83k:53010, Zbl 0477.53056.

[11] J. Erbacher, Reduction of the codimension of an isometric immersion, J. Diff. Geom., 5 (1971), 333-340, MR 44 \#5897, Zbl 0221.53031.

[12] H.B. Lawson, Jr., Lectures on Minimal Submanifolds, Vol. I, Second edition, Mathematics Lecture Series, 9, Publish or Perish, Inc., Wilmington, Del., 1980, MR 82d:53035b, Zbl 0434.53006.

[13] A.M. Li and J.M. Li, An intrinsic rigidity theorem for minimal submanifolds in a sphere, Arch. Math., 58 (1992), 582-594, MR 93b:53050, Zbl 0767.53042.

[14] M. Obata, The Gauss map of immersions of Riemannian manifolds in spaces of constant curvature, J. Diff. Geom., 2 (1968), 217-223, MR 38 \#2705, Zbl 0181.49801.

[15] R. Osserman, Minimal surfaces, Gauss maps, total curvature, eigenvalue estimates, and stability, in 'The Chern Symposium 1979' (Proc. Internat. Sympos., Berkeley, Calif., 1979), 199-227, Springer, New York-Berlin, 1980, MR 82e:53013, Zbl 0457.53001.

Received November 16, 2000.

Department of Mathematics

UNIVERSITY OF IOANNINA

IoANnina 45110, Greece

E-mail address: tvlachos@cc.uoi.gr 